\title{
Barrier Coverage with Airdropped Wireless Sensors
}

\author{
Anwar Saipulla \\ Benyuan Liu \\ Department of Computer Science \\ University of Massachusetts Lowell \\ Lowell, MA 01854 USA \\ \{asaipull, bliu,wang\}@cs.uml.edu
}

Jie Wang

\begin{abstract}
Barrier coverage of a wireless sensor network aims at detecting intruders crossing the network. It provides a viable alternative for monitoring boundaries of battlefields, country borders, coastal lines, and perimeters of critical infrastructures. Early studies on barrier coverage typically assume that sensors are deployed uniformly at random in a large area. This assumption, while theoretically interesting, may be unrealistic in real applications. We take a more realistic approach in this paper. In particular, we consider that sensors are airdropped from an aircraft along its flying route. We note that wind, geographic terrain, and other factors may cause a sensor to land in a location deviating from its targeted landing point with a random offset. Thus, it is more realistic to assume that sensor nodes are distributed with a normal offset along the deployment line. Through extensive simulations, we study how variance of the normal distribution, the number of deployment lines, and the distance between adjacent lines would affect barrier coverage. We then investigate how a strong barrier can be formed efficiently from airdropped sensors and compare it with barrier coverage using uniformly distributed sensors. Our results show that the barrier coverage, with appropriately chosen deployment parameters, can be significantly improved using normally distributed sensors.
\end{abstract}

\section{INTRODUCTION}

Barrier coverage [1] of a wireless sensor network deployed in a region of interest provides a viable mechanism for detecting intruders crossing the region, including boundaries of battlefields, country borders, coastal lines, and parameters of critical infrastructures.

The quality of barrier coverage depends strongly on, among other things, how sensors are deployed. Placing sensors side by side along a straight line across the region of interest is the simplest solution, which is also optimal, because it allows maximum coverage using the minimum number of sensors. However, this strategy is infeasible in many applications because of the large size of the deployment region, the rigid environment constraints, and other factors. On the other hand, dropping sensors from an aircraft or artillery ordinance provides a viable approach to quickly deploying a large number of sensors in a vast geographic region [2]. To deploy sensors in hostile zones or other difficult-to-reach regions, this may be the only feasible deployment strategy.

When sensors are dispersed from an aircraft, environmental factors such as wind and terrain characteristics may cause a sensor to land in a location deviating from its target point with a random offset. This is illustrated in Figure 1. In this paper, we assume that sensors are deployed along straight lines, and the offset of each sensor is a normally distributed random variable. For convenience, we refer to the resulting sensor distribution as line-based normal random offset distribution (LNRO).

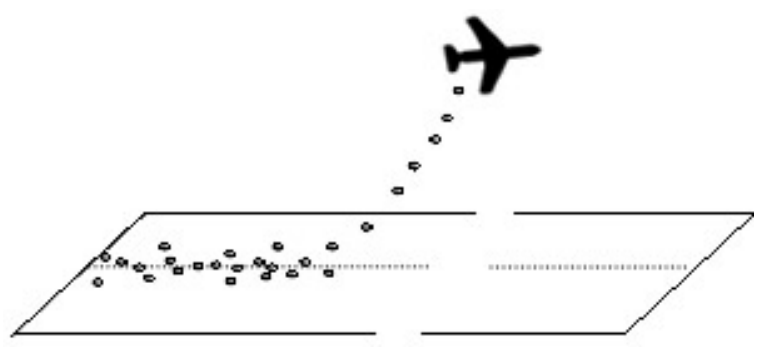

Fig. 1. A line-base deployed wireless sensor network. Actual landing points of sensors will deviate from their targeted locations because of environment factors.

Most of the prior results on barrier coverage are based on the assumption that sensors are distributed uniformly at random in a large area. In the corresponding asymptotic analysis, the sensor locations follow a Poisson point process of a certain density. While this uniform random distribution model may be appropriate for some deployment strategies, it does not capture the sensor distribution under the airdropped deployment strategy, where sensors tend to be concentrated along the deployment lines. Consequently, previous analytical results obtained from 
the uniform random distribution model are no longer applicable to the line-based deployment strategy.

Via extensive simulations, we study how barrier coverage of the line-based deployment strategy depends on the variance of the random landing offsets, the number of deployment lines, and the distance between adjacent lines. We further investigate the efficiency of the line-based deployment strategy by comparing barrier coverage of normally distributed sensors with that of uniformly distributed sensors. Our results show that, with appropriately chosen deployment parameters, normally distributed sensors can provide much better barrier coverage. Our work helps to mend the gap between previous analytical results based on the uniform sensor distribution and realistic line-based deployment strategy, providing important guidelines to sensor deployments for barrier coverage.

The rest of the paper is organized as follows. Section II reviews previous work on the barrier coverage of sensor networks. In Section III, we describe the network model and define barrier coverage. Section IV describes the algorithm to construct barriers and to calculate the quality of barrier coverage. In Section $\mathrm{V}$, we present our simulation results on the barrier coverage of line-based deployed sensor networks. Based on these results, we discuss the implications to the performance and planning of sensor networks for barrier coverage. We conclude the paper in Section VI.

\section{RELATED WORK}

In this section we review the previous research on the barrier coverage of wireless sensor networks. The notion of barrier coverage was first introduced in the context of robotics sensors [1]. The goal of barrier coverage is to detect intruders attempting to cross from one side of a region to the opposite side. Several different barrier coverage measures and the related issues have been studied.

In [11], path coverage is defined and efficient algorithms are proposed to find the maximum breach or support paths between two end points that are least or most likely to be detected by sensors. In [11], the notion of path exposure is introduced to measure the likelihood that an intruder is detected when moving along a given path. A centralized algorithm is proposed to find the minimum exposure paths, where the probability of an intruder being detected is minimized. These path coverage problems are further studied in [9] [14], where efficient distributed algorithms are proposed.
In [10], Liu and Towsley first studied the barrier coverage of two-dimensional plane and two-dimensional strip sensor networks using results in percolation theory. The barrier coverage of a two-dimensional plane network is related to the existence of a giant sensor cluster that percolates the network. For a two dimensional strip network of finite width, it is proved that there always exist crossing paths along which an intruder can cross the strip undetected. Furthermore, the probability that an intruder is detected when crossing a strip is characterized.

In [8], Kumar et al. devised a centralized algorithm to determine whether a region is k-barrier covered, and derived the critical conditions for weak barrier coverage in a randomly deployment sensor network. In [4], Chen et al. devised a localized algorithm that guarantees the detection of intruders whose trajectory is confined to a slice of the belt region of deployment. Recently in [3], Liu et al. derived the critical conditions for strong barrier coverage and devised efficient algorithms to construct strong sensor barriers.

\section{NeTwORK MOdEL}

We assume that sensors are deployed in a twodimensional rectangular area of length $l$ and width $w$, as shown in Figure 2. All sensors are static. They do not move after landing. The location of each sensor in the network is assumed to be known to the node itself using either on-board GPS unit or other localization mechanisms.

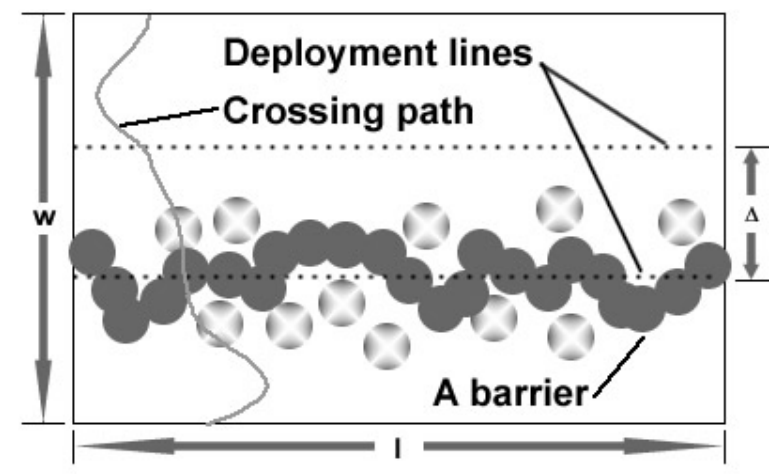

Fig. 2. Sensors are deployed in a rectangular area of $l \times w$. The distance between adjacent deployment lines is $\Delta$.

In the uniform random distribution model, as the name suggests, sensors are independently distributed within the rectangle area uniformly at random. For each sensor, denote its coordinates as $(x, y)$. The probability density 
function (pdf) of the sensor location is:

$$
f(x, y)= \begin{cases}\frac{1}{l w}, & 0 \leq x \leq l, 0 \leq y \leq w, \\ 0, & \text { otherwise }\end{cases}
$$

In the line-based deployment strategy, we assume that there are $m$ evenly spaced horizontal dropping lines along the length of the rectangle. The distance between each pair of adjacent lines is $\Delta$. Denote by $y_{j}^{t}$ the vertical coordinates of the $j^{\prime} t h$ target deployment lines, where $0 \leq j<m$. We have

$$
y_{j}^{t}=y_{0}^{t}+j \Delta .
$$

Along each line, $n$ sensors are to be evenly distributed. The horizontal coordinates of the targeted landing points are

$$
x_{i}^{t}=\frac{l}{n+1} i, 1 \leq i \leq n .
$$

Due to environmental factors such as wind and terrain constraints, the actual landing point of each sensor will deviate from its target by some random offset. Denote the offset of sensor $s_{i}$ in the horizontal and vertical directions by $\delta_{i}^{x}$ and $\delta_{i}^{y}$, respectively. On the $j$-th line, the actual landing point of sensor $s_{i}$ is thus $\left(x_{i}^{t}+\delta_{i}^{x}, y_{j}^{t}+\delta_{i}^{y}\right)$.

We assume that the offsets of the sensors are independent and identically distributed (i.i.d.) with a normal distribution of zero mean and variance $\sigma^{2}$, i.e.,

$$
\delta_{i}^{x}, \delta_{i}^{y} \sim N\left(0, \sigma^{2}\right) .
$$

The sensing model of each sensor follows the widely adopted binary disk model. Each sensor has a sensing range $r$ and can detect any intruders within its sensing range. Two nodes are said to be connected if their sensing areas overlap. In other words, node $s_{i}$ is connected to node $s_{j}$ if and only if $\left|s_{i}-s_{j}\right| \leq 2 r$, where $\left|s_{i}-s_{j}\right|$ represents the Euclidian distance between the two sensor nodes. A sensor barrier is formed by a set of connected sensors that intersect both of the left and right boundaries of the strip. No intruders can cross a sensor barrier without being detected.

A crossing path is a path that connects one side of the region to the opposite side, where the ingress point and the egress point reside on two opposite sides of the region. We assume that the intruders attempt to cross the region along the width of the rectangle area.

The strength of the barrier coverage in a sensor network can be measured by the number of disjoint barriers in the network. A path is said to be $k$-covered if it intercepts at least $k$ distinct sensors. We say that an event occurs with high probability (w.h.p.) if its probability tends to 1 as $n \rightarrow \infty$.
Definition 1: A sensor network is strongly $k$-barrier covered if

$$
P(\text { any crossing path is } k \text {-covered })=1 \text { w.h.p. }
$$

In [8], Kumar et al. introduced the notion of weak barrier coverage, which guarantees to detect intruders moving along congruent crossing paths. But it does not guarantee to detect intruders moving along arbitrary crossing paths as for strong barrier coverage. In the scope of this paper, we will focus on the strong barrier coverage. For convenience, we will simply use barrier coverage to mean strong barrier coverage.

\section{CONSTRUCTING BARRIERS}

We now present the algorithm to compute the number of barriers in a sensor network. We assume that the location of each sensor is obtained by an onboard GPS device or computed using a node localization algorithm. Prior to computation, the location information of all sensors are collected.

We first construct a network flow graph based on the sensor location information, and then compute the maximum flow of the graph. The maximum flow will give the number of barriers in the original sensor network.

\section{Algorithm to Construct Barriers:}

1) Construct a flow graph $G(V, E)$ as follows. Each vertex in $V$ represents a sensor node. For any two vertices $u$ and $v$ in $V$, if their sensing areas overlap, add edge $(u, v)$ in $E$.

2) Construct a weighted directional graph $G^{*}\left(V^{*}, E^{*}\right)$ from $G(V, E)$ as follows. For any node $n_{i}$ in $\mathrm{V}$, add $n_{i}^{\prime}$ and $n_{i}^{\prime \prime}$ to $V^{*}$, and an edge $\left(n_{i}^{\prime}, n_{i}^{\prime \prime}\right)$ to $E^{*}$. If edge $\left(n_{i}, n_{j}\right)$ exists in $E$, add edges $\left(n_{i}^{\prime \prime}, n_{j}^{\prime}\right)$ and $\left(n_{j}^{\prime \prime}, n_{i}^{\prime}\right)$ to $E^{*}$. Add two new vertices $s$ and $d$ into $V^{*}$. For any node $n_{i}$ in $V$, if its sensing range intersects the left boundary of the area, add edge $\left(s, n_{i}^{\prime}\right)$ to $E^{*}$; if its sensing range intersects the right boundary of the area, add edge $\left(n_{i}^{\prime \prime}, d\right)$. The capacity of each edge in $E^{*}$ is set to 1 . For each edge in $E^{*}$, add its reverse edge to $E^{*}$, and set the capacity of this new edge to 0 .

3) Compute the maximum flow from $s$ to $d$ in graph $G^{*}\left(V^{*}, E^{*}\right)$ using a standard algorithm (e.g., FordFulkerson, Edmond-Karp, or the relabel-to-front algorithms [5]). The maximum flow of the graph is the number of disjoint barriers in the original sensor network.

Note that in Step 2, each sensor corresponds to two graph nodes with one edge between them. This is to 
guarantee that each sensor node is only used once in barrier construction. In Step 3, each augmenting path forms a barrier in the sensor network.
(0)

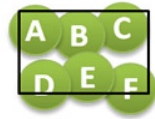

(2)

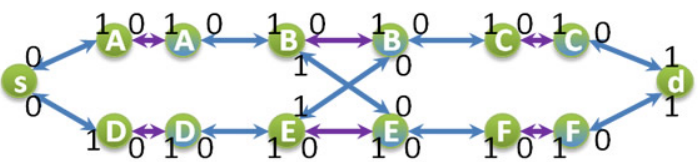

(3)

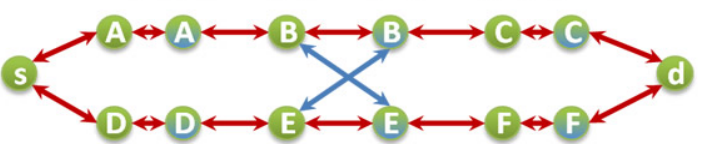

Fig. 3. Steps of constructing barriers in a sensor network of six sensors. Each sub-picture represents the result of one step in the algorithm above.

Figure 3 illustrate the steps of the algorithm for a small sensor network of six nodes. This network have 2 disjoint barriers.

\section{Simulation Results}

We carry out a series of simulations to study the barrier coverage of the line-based deployment strategy. Unless indicated otherwise, the rectangle's length is set to $l=10,000$ meters, and the sensing range of each sensor to $r=10$ meters. Each point in the plots represents the average value of 1,000 experiments.

\section{A. Line-based Normal Distribution vs. Uniform Distri- bution}

In the case of uniform distribution, each sensor has the equal likelihood to be located at any point in the rectangle. Thus, the sensors are spread out rather evenly in the area. It has been proved in [3] that in the asymptotic case, barriers exist if and only if the width of the rectangle is larger than the logarithm of the length and at the same time the sensor density is greater than some critical value.

In the line-based deployment strategy with normally distributed random offsets, sensors are concentrated along the deployment line. The node density in the vertical direction forms a "bell" curve whose shape is determined by the variance of the normal distribution. Figure 4 illustrates the deployment layouts of the uniform distribution and line-based normal distribution, and the corresponding sensor densities in the vertical direction.

Compared to the uniform distribution, sensors are concentrated along deployment lines in the line-based
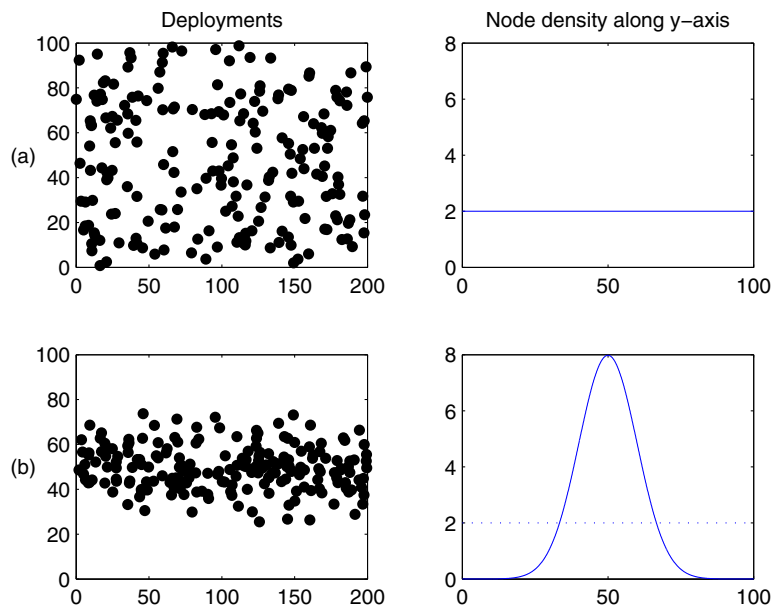

Fig. 4. 200 Sensors deployed based on uniform distribution and line-based normal distribution, and the corresponding sensor density on y-axis. (a)Uniform distribution, $\lambda=0.02$; (b)Single-line LNRO, $\sigma=10$.

deployment strategy, forming line clusters, which may provide a better chance for barriers to be formed. To compare the barrier coverage of the line-based normal distribution with that of the uniform distribution, we note that $99.7 \%$ of the sensors fall within the distance of $3 \sigma$ from the deployment line in LNRO, and so we choose the width of the rectangle for the uniform distribution to be $6 \sigma$ for comparison.

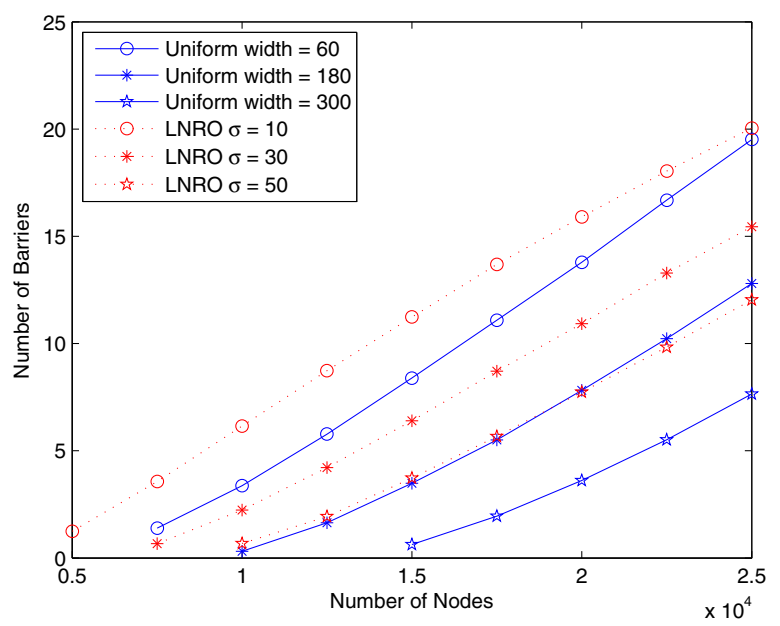

Fig. 5. Comparison of the barrier coverage of line-based normal distribution to uniform distribution.

Figure 5 plots the number of barriers of the two distributions for three different scenarios. We compare the barrier coverage of LNRO with $\sigma=10,30$, and 50, to those of the uniform distribution of width 60,180 , 
and 300, respectively. It can be observed that the LNRO consistently outperforms the uniform distribution.

\section{B. Single-Line Deployment}

In this part of the experiments we consider a singleline sensor deployment scenario and focus our study on the effect of offset variance $\left(\sigma^{2}\right)$ on the barrier coverage.

As sensors are deployed along a line with normally distributed random offsets, the variance of the random offsets characterizes how concentrated the sensors are located relative to the deployment line. As the variance increases, the sensors will be more spread out in a larger region. We expect this will result in a smaller number of barriers.

We deploy sensor along the central line of a rectangle area of 10,000 meters by 1,000 meters. The offset variance $\left(\sigma^{2}\right)$ is varied from 0 to 130 by an incremental interval of 1 . The width of the field is set large enough to ensure that almost all of the sensors fall into the rectangle for the range of the offset variances. Figure 6 plots the number of barriers as a function of the offset variance for six different choices of number of sensors, that is, $n$ $=5,000,7,500,10,000,125,000,150,000$, and 175,000, respectively.

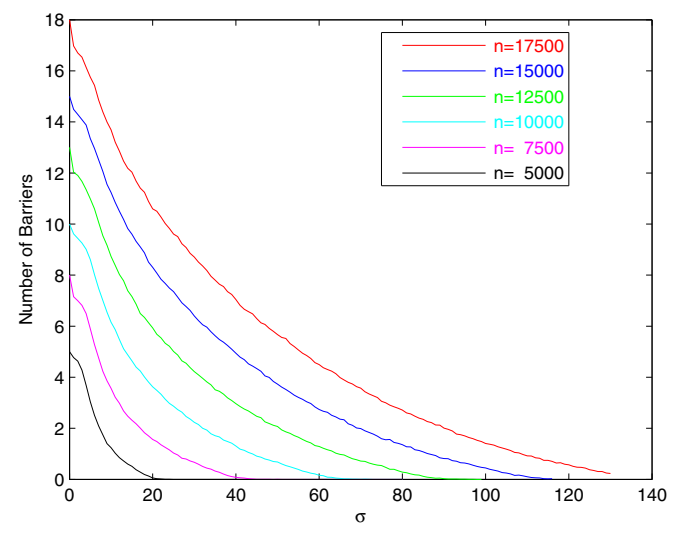

Fig. 6. Barrier Coverage of LNRO distributed sensors. For a fixed number of sensors, the number of barriers decreases as variance $\sigma$ increases.

It can be observed that for a fixed total number of sensors, the number of barriers decreases as the variance of the normal distribution increases. This is consistent with what we have expected.

For a fixed offset variance $\left(\sigma^{2}\right)$, the sensor distribution around the deployed line is kept constant. As more sensors are deployed, the number of barriers shows a linear increase of the number of sensors. This is shown in Figure 7.

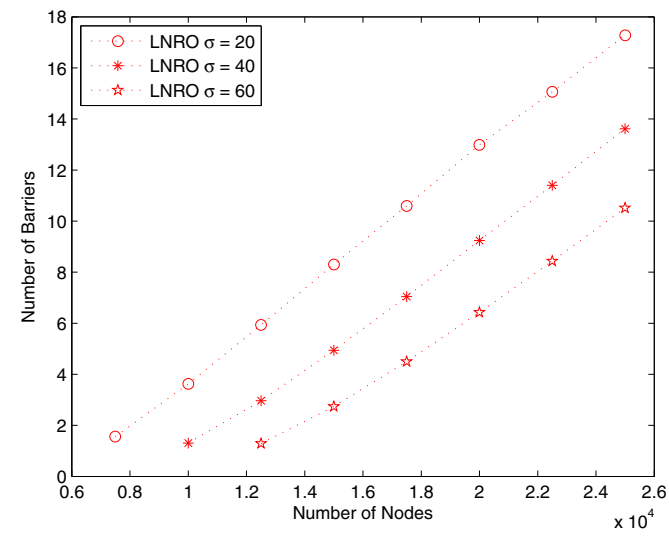

Fig. 7. Barrier Coverage of LNRO distributed sensors as a function of the number of sensors.

\section{Multiple-Line Deployment}

In some applications, sensors may be airdropped along multiple lines, to provide robustness and multiple lines of defense in the deployed region. In this part, we consider a two-line deployment scenario to focus on the effect of two deployment parameters: the random offset variance $\left(\sigma^{2}\right)$ and the distance between the two deployment lines $(\Delta)$. Figure 8 depicts two-line deployment scenarios for 200 nodes with two different $\Delta$ values.
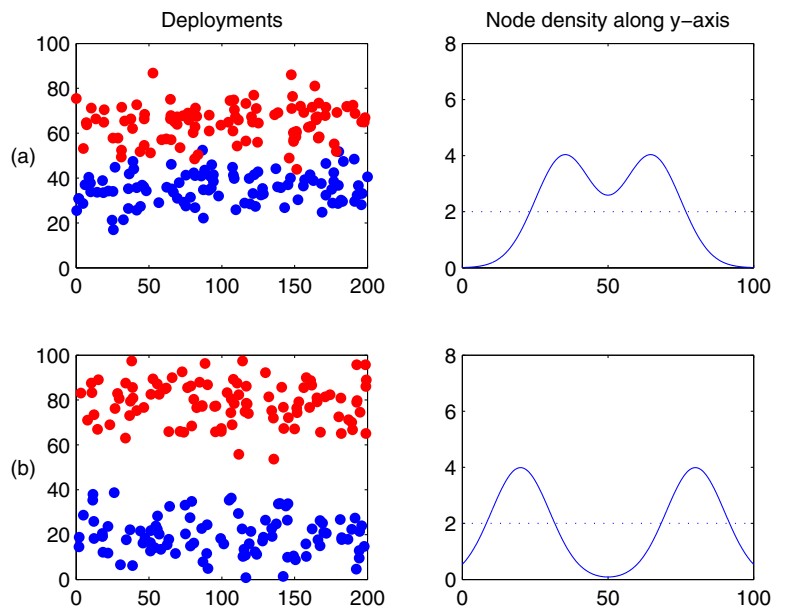

Fig. 8. Sensor distributions of two-line deployment scenarios, and the corresponding sensor densities in the vertical direction. (a) $\Delta=$ 30 meters ; (b) $\Delta=60$ meters.

In the following experiments, a total number of 10, 000 sensors are deployed along two lines of distance $\Delta$ in a rectangle area of length $l=10,000$ meters, according to the LNRO distribution. The sensors are equally divided into the two deployment lines. The width 
of the field is chosen to be large enough so that all the nodes fall into the rectangle in the simulations. We consider four different offset variance values: $\sigma=5,10$, 15 , and 30 .

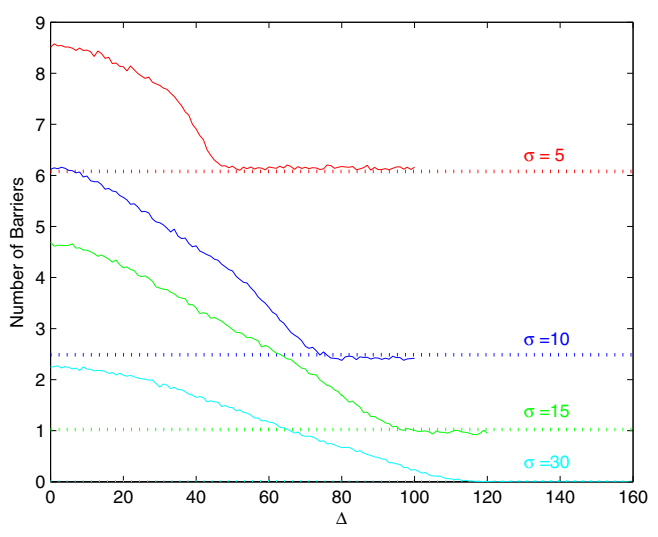

Fig. 9. Barrier coverage of a two-line deployed sensor network.

Figure 9 plots the number of barriers as a function of $\Delta$ for the four offset variance values. The $\Delta=0$ cases correspond to the scenario where the two deployment lines coincides with each other. Thus the effect is equivalent to the case when the same number of sensors are deployed along a single line. The number of barriers decreases as the distance $\Delta$ increases. This can be explained as follows. When the two lines are close to each other, there is a significant overlap between the two groups of sensors, as shown in Figure 8(a). A sensor in one group can connect to sensors in its own group or sensors in the its neighboring group. As $\Delta$ increases, i.e., as the two deployment lines become farther apart from each other, there is less overlap between the two groups of sensors, resulting in a degraded barrier coverage.

In particular, when $\Delta$ increases to roughly $(6 \sigma+2 r)$, the number of barriers levels off to about twice that of a single line deployment with half of the sensors (dashed lines). At this distance, there is almost no overlap between the two groups of sensors, and the probability of forming barriers using sensors from both groups diminishes.

\section{Deployment Implications}

Our simulation results have important implications to the performance and planning of sensor networks for barrier coverage. In particular, if other deployment parameters are kept the same, a smaller variance results in a larger number of barriers. Hence it is important to reduce the landing offset variance if at all possible. The variance may be reduced, for example, by having the aircraft fly closer to the ground to minimize the effects of the environment factors such as wind. The weight and packaging of sensors may also play a vital role in reducing the variance.

Our results show that the number of barriers increases proportionally to the number of sensors. Thus, when small variance is hard to achieve due to weather conditions or hostile environments, increasing the number of sensors is an effective approach to achieving the desired barrier coverage.

When sensors are deployed along multiple lines, it is desirable to keep the lines close to each other in order to take advantage of the "overlapping effect" between adjacent groups of sensors. Note that this benefit diminishes when the distance between adjacent lines becomes larger than $6 \sigma+2 r$.

\section{CONCLUSION}

We study the barrier coverage of airdropped wireless sensors. Due to the environment factors such as wind and geographic terrain, the sensors will be scattered around the deployment line with some random offsets. In this paper we model the offsets as normally distributed random variables. Via extensive simulations, we investigate how barrier coverage relies on variance of the normally distributed random offset, the number of deployment lines, and the distance between adjacent lines. Our results show that in a single-line LNRO deployment, if we deploy the same number of sensors in the same region, the number of barriers is significantly larger than that of a uniform sensor distribution, as sensors are concentrated along the given line. We also show that the variance of the random landing offsets has a significant impact on the barrier coverage. In the case of multiple-line deployment, sensors in different groups may overlap and connect together, resulting in an improved barrier coverage.

\section{ACKNOWLEDGEMENT}

We thank the anonymous reviewers for their helpful comments. Benyuan Liu was partly supported by the National Science Foundation under grant CNS-0721626. Jie Wang was supported in part by NSF under grants CNS-0709001 and CCF-0830314.

\section{REFERENCES}

[1] D. Gage. Command control for many-robot systems. In Proc. of the Nineteenth Annual AUVS Technical Symposium (AUVS-92), 1992.

[2] Tracking vehicles with a UAV-delivered sensor network. Avaliable at http://robotics.eecs.berkeley.edu/ pister/29Palms0103/. 
[3] B. Liu, O. Dousse, J. Wang and A. Saipulla Strong Barrier Coverage of Wireless Sensor Networks. In Proc. ACM Mobihoc, 2008.

[4] A. Chen, S. Kumar, and T.-H. Lai. Designing localized algorithms for barrier coverage. In Proceedings of ACM Mobicom, 2007.

[5] T. H. Cormen, C. E. Leiserson, R. L. Rivest, and C. Stein. Introduction to Algorithms (Second Edition). MIT Press and McGraw-Hill, 2001.

[6] M. Franceschetti, O. Dousse, D. Tse, and P. Thiran. Closing the gap in the capacity of random wireless networks. In Proc. of Information Theory Symposium (ISIT), 2004.

[7] G. R. Grimmett. Percolation. Springer, 1999.

[8] S. Kumar, T. H. Lai, and A. Arora. Barrier coverage with wireless sensors. In Proc. ACM Mobicom, 2005.

[9] X.-Y. Li, P.-J. Wan, and O. Frieder. Coverage in wireless ad-hoc sensor networks. IEEE Transactions on Computers, 52(6):753763, June 2003.

[10] B. Liu and D. Towsley. A study on the coverage of large-scale sensor networks. In The 1st IEEE International Conference on Mobile Ad-hoc and Sensor Systems, 2004.

[11] S. Meguerdichian, F. Koushanfar, M. Potkonjak, and M. B. Srivastava. Coverage problems in wireless ad-hoc sensor networks. In Proc. IEEE Infocom, pages 1380-1387, 2001.

[12] S. Meguerdichian, F. Koushanfar, G. Qu, and M. Potkonjak. Exposure in wireless ad-hoc sensor networks. In ACM Mobile Computing and Networking, pages 139-150, 2001.

[13] A. Schrijver. Combinatorial Optimization. Springer, 2003.

[14] G. Veltri, Q. Huang, G. Qu, and M. Potkonjak. Minimal and maximal exposure path algorithms for wireless embedded sensor networks. In Proc. of ACM Sensys, 2003. 